\title{
Abnormal skin changes and unilateral vision loss after a tuberculin skin test
}

\author{
Sumana C. Naidu BHSc MD(C), Alexander J. Kaplan MD, Nick Daneman MD MSc, Arun N.E. Sundaram MD MSc, \\ Radha P. Kohly MD PhD
}

Cite as: CMAJ 2021 November 29;193:E1811-4. doi: 10.1503/cmaj.211021

A healthy 20-year-old woman had a tuberculin skin test (TST), as required by her employer. Over 2 days, she developed a necrotizing reaction with a $1 \mathrm{~cm}$ central erosion surrounded by about $6 \mathrm{~cm}$ of induration with hyperpigmentation at the site of the TST (Figure 1). Six days later, she developed painless vision loss in the left eye, followed by a leftsided headache and periorbital swelling.

She was of Jamaican descent and born in Canada, had never received the Bacillus Calmette-Guérin vaccine, had no known personal or family history of tuberculosis (TB), and had not recently travelled to TB-endemic countries. Her medical history was unremarkable with no risk factors for TB, and she had had no symptoms before the TST. Her mother, however, was a personal support worker; health care workers are potential sources of TB exposure.

Ocular assessment showed that the patient's visual acuity was near normal (20/20-3) in the right eye but severely reduced (counting fingers only at 2 feet $[0.5 \mathrm{~m}]$ ) in the left eye. Ocular examination showed that her right eye was normal. In her left eye, there was a relative afferent pupillary defect, with trace anterior segment cells, trace vitritis, florid optic disc edema, peripapillary hemorrhages and intraretinal fluid (Figure 2). Optical coherence tomography of her right eye was normal, but her left eye showed peripapillary intraretinal and subretinal fluid extending into the fovea (Figure 3).

Given the positive TST and unilateral panuveitis, our working differential diagnosis included a hypersensitivity reaction to the TST reagent, with or without reactivation of latent TB. The patient said she had no systemic or respiratory symptoms, and results of extensive serologic investigations - including a complete blood count and angiotensin-converting enzyme test - were normal, ruling out differentials such as sarcoidosis, and lowering clinical suspicion of lymphoma. Results of tests for syphilis and HIV were negative. Given the low pre-test probability of latent TB, we considered a nonspecific hypersensitivity reaction to the TST reagent to be just as likely as a true positive TST.

We ordered a QuantiFERON-TB Gold Plus (QFT-Plus) test to confirm the TST result. QFT-Plus is an interferon- $\gamma$ release assay blood test that has greater sensitivity than the TST. ${ }^{1}$ While we

\section{Key points}

- The incidence of active tuberculosis (TB) in Canada is about 4.8 per 100000 people, and latent TB affects more than 1.5 million people, in about $5 \%-10 \%$ of whom the disease will reactivate.

- Tuberculin skin test (TST) is performed to diagnose latent TB, in the absence of clinical symptoms, and may cause an immune reaction that can, uncommonly, reactivate latent TB.

- With any systemic complication after TST - including uncommon skin changes at the TST site, such as necrosis and hyperpigmentation, and reduced visual acuity - a diagnosis of TB should be considered, even in patients with low pre-test probability.

waited for the QFT-Plus test results, our patient's vision deteriorated, and we admitted her to hospital.

As the patient had presented with uveitis and deteriorating vision, we performed a lumbar puncture to rule out infectious and inflammatory etiology; spinal fluid analysis was normal. Contrast- enhanced magnetic resonance imaging of her brain and orbits showed no optic nerve sheath or leptomeningeal enhancement, suggesting uveitis-related inflammatory papillitis as the cause of the left optic disc edema. After a chest radiograph showed a prominent right hilum, a computerized tomography scan demonstrated heterogeneous-appearing, enlarged mediastinal, right hilar and interlobar lymph nodes with minor tree-inbud changes in the right middle lobe (Figure 4).

We started the patient on intravenous methylprednisolone with the presumptive diagnosis of a hypersensitivity reaction to the TST. She then underwent endobronchial ultrasoundguided transbronchial needle aspiration of station 4R, 7 and $11 \mathrm{R}$ lymph nodes. Cytology from the station 7 node showed necrosis, and stains were negative for fungal organisms and acid-fast bacilli.

Two days after treatment, the patient's visual acuity improved from counting fingers to $20 / 100-2$ in the left eye. Her headache subsided, periorbital swelling resolved, and she transitioned to oral prednisone. One week after starting oral prednisone, she was found to have new, subretinal creamy yellow lesions throughout the fundus (Figure 5). 


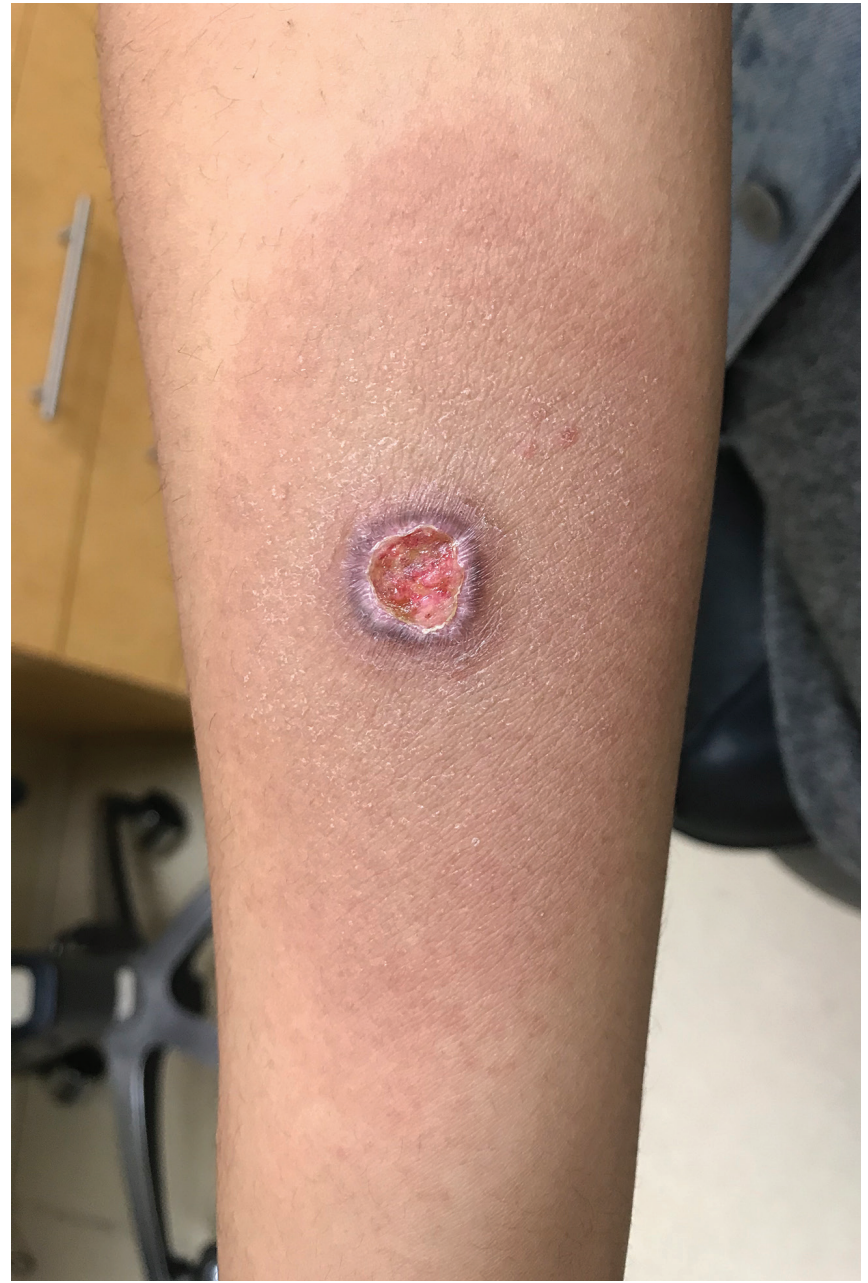

Figure 1: Forearm of a 20-year-old woman, showing a necrotizing reaction to a tuberculin skin test with a $1-\mathrm{cm}$ central erosion surrounded by about $6 \mathrm{~cm}$ of induration with hyperpigmentation.

We then received the results of outstanding investigations; her QFT-Plus was significantly positive with a CD4- and CD8positive shift (TB1 antigen - nil $=6.67 \mathrm{IU} / \mathrm{mL}$, TB2 antigen - nil $=$ $7.66 \mathrm{IU} / \mathrm{mL}$, with a positive cut-off of either value $\geq 0.35 \mathrm{IU} / \mathrm{mL}]$ ) and the lymph node culture was positive for Mycobacterium tuberculosis. We therefore considered the most likely diagnosis to be a hypersensitivity reaction to the TST with reactivation of latent TB. We started her on anti-TB medication: isoniazid $300 \mathrm{mg} / \mathrm{d}$, rifampin $600 \mathrm{mg} / \mathrm{d}$, pyrazinamide $1500 \mathrm{mg} / \mathrm{d}$, and vita$\min B_{6} 25 \mathrm{mg} / \mathrm{d}$. We increased the oral prednisone to $60 \mathrm{mg} / \mathrm{d}$ with a slow tapering schedule. Two weeks after the patient started anti-TB therapy, her choroidal lesions, optic disc edema and macular edema resolved.

\section{Discussion}

Tuberculosis is an airborne infection caused by M. tuberculosis. It is spread through droplets expelled from the airways of individuals with active infection. ${ }^{1}$ When these droplets are inhaled, they lodge in the lung alveoli and begin to multiply, leading to a host immune response. The balance between host immunity and bac-

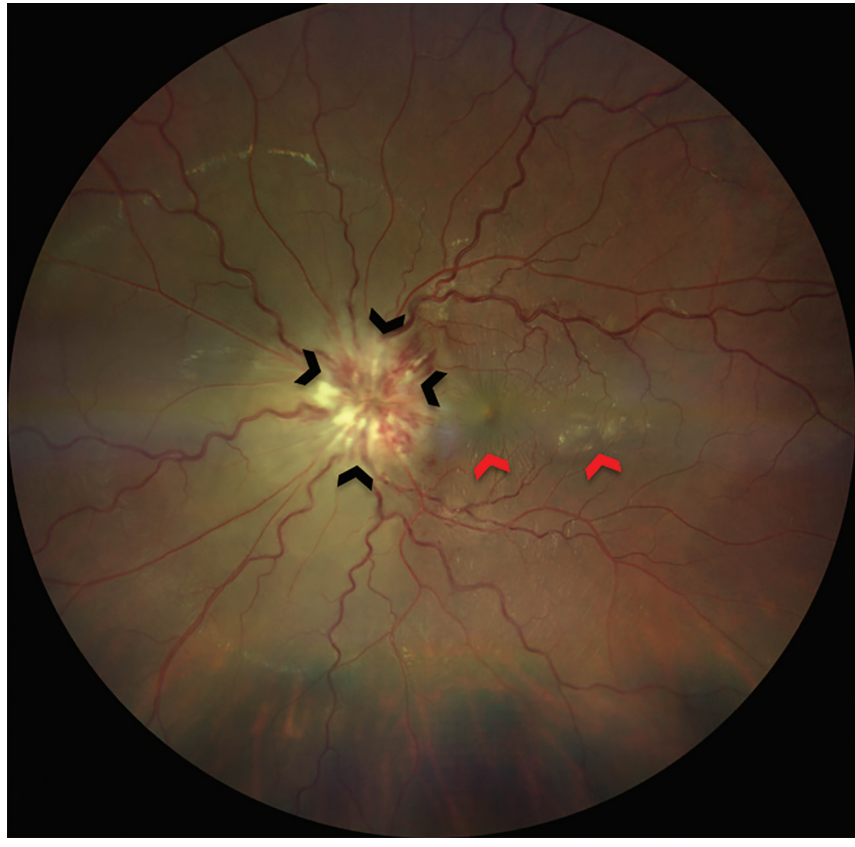

Figure 2: Fundus image of the patient's left eye at time of presentation showing optic disc edema with peripapillary hemorrhages (black arrows) and intraretinal and subretinal fluid into the macula (red arrows).

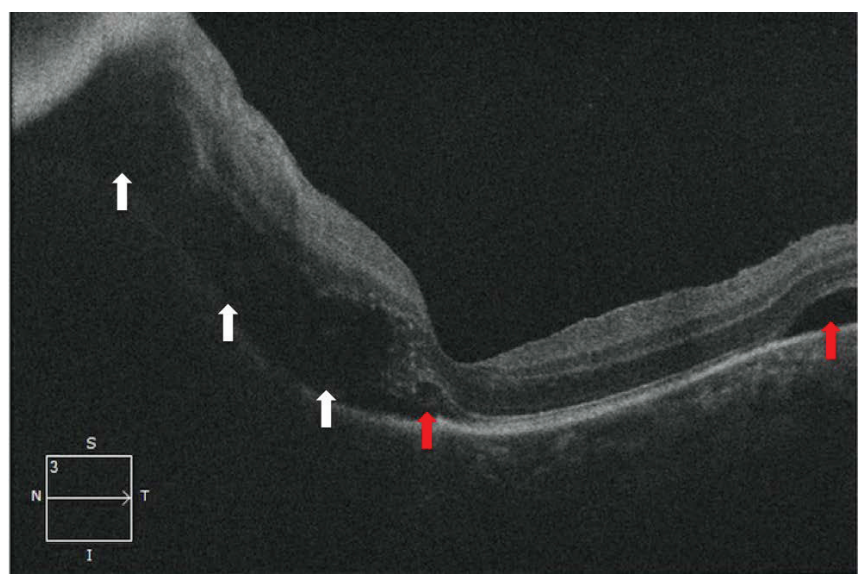

Figure 3: Optical coherence tomography of the patient's left eye at time of presentation, showing peripapillary intraretinal and possibly subretinal fluid (white arrows), and subretinal fluid extending into the fovea (red arrows).

illary multiplication determines the outcome of infection. Three potential outcomes follow infection: symptomatic active TB, latent TB and delayed reactivation of latent TB. ${ }^{1} \mathrm{~A}$ delayed hypersensitivity reaction may also occur after infection. ${ }^{2}$ In Canada, the incidence of active TB is about 4.8 per 100000 people, and more than 1.5 million individuals live with latent TB, which has a lifetime reactivation risk of $5 \%-10 \%$. $^{3}$

Active TB follows an inadequate immune response to $M$. tuberculosis, and presents with a range of symptoms that include productive cough, hemoptysis, fever, weight loss, night sweats and lymphadenopathy. Extrapulmonary manifestations occur in the bowel, spine (Pott disease), or central nervous system (tubercular meningitis), among others. ${ }^{1,4}$ Latent TB, which is an asymptomatic clinical state, occurs when an individual is 

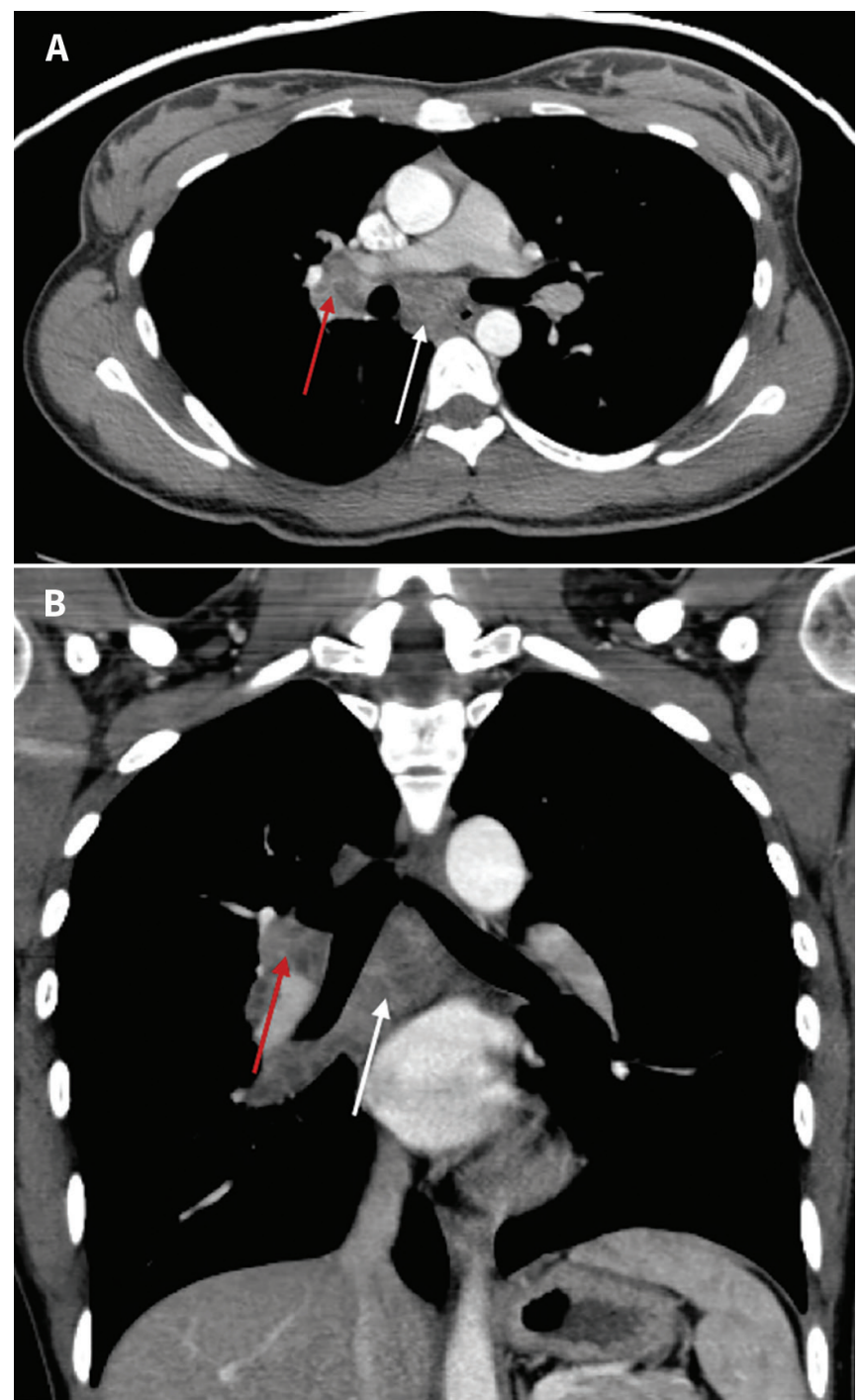

Figure 4: Axial (A) and coronal (B) views of a contrast-enhanced computerized tomography scan of the patient's chest, showing enlarged, heterogeneous mediastinal (white arrow) and right interlobar (red arrow) lymph nodes.

infected with TB but mounts an effective immune response to contain bacillary multiplication. It covers a broad range of clinical states, from dormant bacilli to subclinical infection with actively replicating bacteria. Because of the containment of bacillary multiplication, latent TB is not diagnosed based on the presence of bacilli. ${ }^{1}$

Reactivation of latent TB can occur years after the primary infection, when the balance between host immunity and bacillary multiplication is disturbed, leading to a loss of immune control and a shift toward reactivation and multiplication of bacilli. Risk factors for reactivation include immunosuppression, alcohol consumption, smoking, malignancy, diabetes and renal failure. ${ }^{1}$

Delayed hypersensitivity reactions may be caused by an exaggerated immune response to mycobacterial antigens. This can occur in active or latent TB infection. Delayed hypersensitivity reactions are most notably reported with ocular manifestations of TB, such as Eales disease. ${ }^{2}$

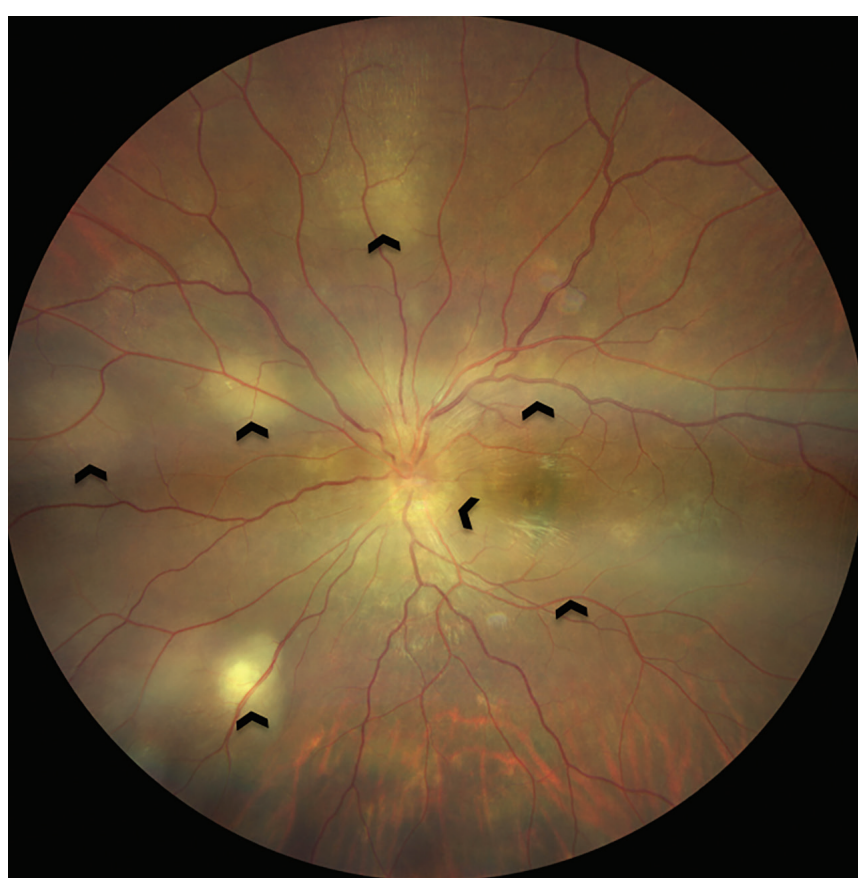

Figure 5: Fundus photo of the patient's left eye taken 1 month after presentation, showing deep multifocal choroidal whitish lesions (arrows) after reinitiation of oral steroids.

In Canada, latent TB is diagnosed with a positive TST or QFT-Plus, in the absence of clinical symptoms. The TST or QFTPlus tests measure the immune response to $M$. tuberculosis antigens. ${ }^{1}$ In the TST, a small amount of purified protein derivative of $M$. tuberculosis is injected intradermally, whereas the QFT-Plus is a blood test. With TST, in someone with latent TB, there is a delayed type IV hypersensitivity reaction that peaks within 2-3 days when the test is read, while the QFT-Plus can be completed in 24 hours., ${ }^{5,6}$ The TST has cross-reactivity in those who have received the Bacillus Calmette-Guérin vaccine; therefore, QFT-Plus is the preferred testing method for these patients. ${ }^{7}$ Neither test can differentiate between latent or active TB.

Four cases have been reported in the literature on ocular reactions to TST, and none of the patients had any risk factors for TB. The most recent case reported bilateral multifocal choroidal tubercles after a TST, and a T-SPOT.TB test was negative. ${ }^{8}$ No systemic features of TB were found and no tissue samples were cultured; the patient responded rapidly to steroid and active antituberculosis therapy. In another case, a hypersensitivity reaction with bilateral serous retinal detachments without evidence of choroidal tubercles after a TST was reported. ${ }^{9}$ The patient responded to steroid therapy and a latent TB regimen. Both patients had almost full recovery of vision.

Our patient had a florid TST reaction, a unilateral granulomatous panuveitis, a positive QFT-Plus, and a culture-positive mediastinal lymph node after the TST reaction, suggesting the presence of active TB. ${ }^{7}$ We hypothesize that the TST caused a systemic hypersensitivity reaction and reactivated latent TB. The dramatic forearm ulceration after the TST is highly indicative of an immune-mediated response. The subsequent activation of 
ocular tubercular granulomas, and the presence of biopsy-positive TB chest lymph nodes, suggests reactivation of latent TB.

We cannot rule out preceding subclinical active $T B$, as the patient may have been in a subclinical disease state, with actively replicating bacteria in the absence of clinical symptoms and with no causal relation to the TST. This is less likely, however, as the patient was asymptomatic and had had no known recent exposure to anyone with active TB.

We report an uncommon but important complication that physicians should be aware of when patients present with ocular or systemic complaints after a TST.

\section{References}

1. Gideon HP, Flynn JL. Latent tuberculosis: What the host "sees"? Immunol Res 2011;50:202-12.

2. Sridharan S, Biswas J. Ocular tuberculosis: an update. Expert Rev Ophthalmol 2007;2:845-60.

3. Cohen A, Mathiasen VD, Schön T, et al. The global prevalence of latent tuberculosis: a systematic review and meta-analysis. Eur Respir J 2019;54:1900655.

4. Jilani TN, Avula A, Gondal AZ, et al. Active tuberculosis. StatPearls 2020 Nov. 20. [last updated 2021 Aug. 6].

5. Essue BM, Milinkovic D, Birch S. Better data to drive more effective care for people with latent tuberculosis infection in Canada. CMAJ 2018;190:E700-1.

6. Youssef E, Wooltorton E. Serious allergic reactions following tuberculin skin tests. CMAJ 2005;173:34. doi: 10.1503/cmaj.050710.

7. Kiazyk S, Ball TB. Tuberculosis (TB): latent tuberculosis infection: an overview. Can Commun Dis Rep 2017;43:62-6.

8. Zhao H, Peng X. Tuberculin skin testing induces bilateral tuberculous uveitis in a junior high school student. Ocul Immunol Inflamm 2020;28:1-3.

9. Burgoyne CF, Verstraeten TC, Friberg TR. Tuberculin skin-test-induced uveitis in the absence of tuberculosis. Graefes Arch Clin Exp Ophthalmol 1991;229:232-6.
Competing interests: Sumana Naidu reports being the Research and Evaluation Lead of the Student-Senior Isolation Prevention Partnership. Alexander Kaplan reports receiving payments or honoraria from AbbVie Medical and Bayer. No other competing interests were declared.

This article has been peer reviewed.

The authors have obtained patient consent.

Affiliations: Temerty Faculty of Medicine (Naidu); Department of Ophthalmology and Vision Sciences (Kaplan, Sundaram, Kohly), University of Toronto; Kensington Eye Institute (Kaplan); Department of Medicine, Division of Infectious Diseases (Daneman), and of Medicine, Division of Neurology (Sundaram), Sunnybrook Health Sciences Centre, University of Toronto, Toronto, Ont.

Contributors: All of the authors contributed to the conception and design of the work. Sumana Naidu and Radha Kohly drafted the manuscript. All of the authors revised it critically for important intellectual content, gave final approval of the version to be published and agreed to be accountable for all aspects of the work.

Content licence: This is an Open Access article distributed in accordance with the terms of the Creative Commons Attribution (CC BY-NCND 4.0) licence, which permits use, distribution and reproduction in any medium, provided that the original publication is properly cited, the use is noncommercial (i.e., research or educational use), and no modifications or adaptations are made. See: https://creativecommons.org/ licenses/by-nc-nd/4.0/

Acknowledgements: The authors acknowledge Drs. Christine McDonald and Kaveh Shojania at the Sunnybrook Health Sciences Centre, University of Toronto, for their time and expertise in the management of this case and reviewing the manuscript.

Correspondence to: Radha Kohly, Radha.kohly@utoronto.ca 